\title{
SERVICIO AMBIENTAL DE CAPTURA DE CARBONO DE LOS BOFEDALES DEL CENTRO POBLADO ALTO PERÚ, TACNA
}

\author{
ENVIRONMENTAL CARBON CAPTURE SERVICE OF THE WETLAND OF \\ THE ALTO PERU POPULATED CENTER, TACNA
}

Diego Abel Sequeiros Abarca ${ }^{1}$ José Oswaldo Cazorla Galdos²

Información del artículo:

Recibido: 31/082019

Aceptado: 25/09/2020

\begin{abstract}
${ }^{1}$ Escuela de ingeniería ambiental, Universidad Privada de Tacna, Perú ${ }^{2}$ Docente de escuela de Ingeniería Ambiental, Universidad Privada de Tacna, Perú E-mail: ${ }^{1} \mathrm{dsa}$ _30@hotmail.com, ${ }^{2}$ jcazorlag@yahoo.es
\end{abstract}




\section{Resumen}

La evaluación del servicio ambiental de captura de carbono fue realizado en el Centro Poblado Alto Perú, Región de Tacna, de septiembre a diciembre del 2019. El trabajo empezó con la recopilación de información del área de estudio, incluyendo la descarga de imágenes del satélite Landsat 8, con el fin de calcular el Índice de Vegetación de Diferencia Normalizada (NDVI) y así dividir el área de estudio según el estado de conservación de la cobertura vegetal. Posteriormente se procedió a realizar la recolección de las muestras en campo, siguiendo la metodología del Centro Mundial Agroforestal (2009) recolectando tres tipos de muestras; cobertura vegetal, turba/necromasa y suelo orgánico con el fin de calcular el carbono orgánico total para cada tipo de muestra. Finalmente los análisis de laboratorio sirvieron para la determinación de la fracción de carbono y la densidad aparente del suelo, cuyos datos fueron utilizados para el stock de carbono total. Los resultados indican que el carbono total es 157,18 Tn $\mathrm{C} / \mathrm{Ha}$, demostrándose el alto potencial que tienen los bofedales como aporte al servicio ambiental de captura de carbono; y siendo necesario implementar y reforzar los programas de conservación.

Palabras Claves: Carbono total; Servicio ambiental; bofedales altoandinos.

\section{Abstract}

The evaluation of the environmental carbon capture service was carried out in the Alto Peru populated center, Tacna Region, from September to December 2019. Work began with collecting information from the study area, including downloading images from the Landsat 8 satellite, in order to calculate the Normalized Difference Vegetation Index (NDVI) and thus divide the study area according to the conservation status of plant cover. Subsequently, the samples were collected in the field, following the methodology of the World Agroforestry Centre (2009) collecting three types of samples; plant cover, peat/necromasse and organic soil in order to calculate the total organic carbon for each sample type. Finally, laboratory analyses were used for the determination of the carbon fraction and apparent density of the soil, the data of which were used for the total carbon stock. The results indicate that the total carbon is 157.18 Tn $\mathrm{C} / \mathrm{Ha}$, demonstrating the high potential of wetland as a contribution to the environmental carbon capture service; and it is necessary to implement and strengthen conservation programmes.

Keywords: Total carbon; Environmental service; high Andean bofedales. 


\section{Introducción}

Los bofedales son formaciones vegetativas ubicadas en las zonas altoandinas que generalmente ocupan terrenos inmediatos a lagunas o aguas de corriente lenta, en ellas el suelo está saturado en agua estacional o permanentemente, en estos sitios semipantanosos se desarrolla una vegetación hidrófila siempre verde. (Flores et al., 2005), dichos ecosistemas, son esenciales para la adaptación de muchas comunidades humanas al cambio climático y también, particularmente sensibles a este fenómeno (Franco et al., 2012), además, los bofedales prestan diversos servicios ecosistémicos; se consideran sistemas claves en las dinámicas del ciclo del carbono a nivel global. Esto se debe a que son importantes sumideros de carbono, presentan altas tasas de emisión de metano $(\mathrm{CH} 4)$ y un alto potencial de secuestro de $\mathrm{CO} 2$ mediante la formación de turberas, deposición de sedimentos, y en la producción de biomasa de las plantas (Arellano et al., 2013). El centro poblado de Alto Perú, se encuentra ubicado en el sitio de protección prioritario denominado Alto Perú - Tripartito. Los cuales son claves para la conservación de especies y ecosistemas frágiles. El principal problema que afecta los bofedales del Centro Poblado de Alto Perú es que no cuentan con un eficiente tratamiento de aguas residuales domésticas, cuyos efluentes son vertidos a los cuerpos de agua, ubicados en los bofedales circundantes a dicho centro poblado, ocasionando así un desbalance por el incremento de nutrientes y alterándose así los niveles tróficos.

Con lo planteado anteriormente, es necesario generar nuevos conocimientos que nos permitan valorar los servicios ecosistémicos que nos brindan las diferentes formaciones vegetales, como son los bofedales, para así facilitar la toma de decisiones sobre la conservación y uso eficiente de los recursos

\section{Objetivos}

Estimar el carbono capturado como parte del servicio ambiental de regulación en los bofedales del Centro Poblado Alto Perú.

\section{Metodología}

\section{Determinación de área de estudio}

La investigación es de tipo descriptivo, la población identificada son los bofedales del Centro Poblado Alto Perú, que a su vez se localiza en el departamento de Tacna, que posee un área aproximada de 254,39 ha, la muestra se identificó como la cobertura vegetal, turba/necromasa y el suelo, según López (2015) entre la cobertura vegetal se encuentran las especies antes mencionadas de flora representativa de los bofedales del sur del Perú; Distichia muscoides, Oxychloe andina y Aciachne

pulvinata. Por otra parte, según el Plan Maestro ACRVM (2012) entre las especies almohadilladas predominantes, se encuentran; Distichia muscoides, Oxychloe andina y Plantago rigida. Para las 
muestras bajo la cobertura, se divide entre la capa inicial raíz y/o necromasa a $10 \mathrm{~cm}$ de profundidad desde la superficie y el suelo orgánico; mayor a $30 \mathrm{~cm}$ de profundidad.

Para poder acceder a la zona de estudio, se usó la carretera Tacna - Collpa - La Paz, una vez llegado al sector de paso de los vientos la carretera asfaltada se convierte de trocha hasta alcanzar el Centro Poblado, Alto Perú que se encuentra en las coordenadas UTM: 4230772 este y 8055920 sur a aproximadamente 4200 m.s.n.m., tal como se puede apreciar en la figura 1.

\section{Figura 1}

Ubicación del centro poblado de alto Perú en el Distrito de Palca.

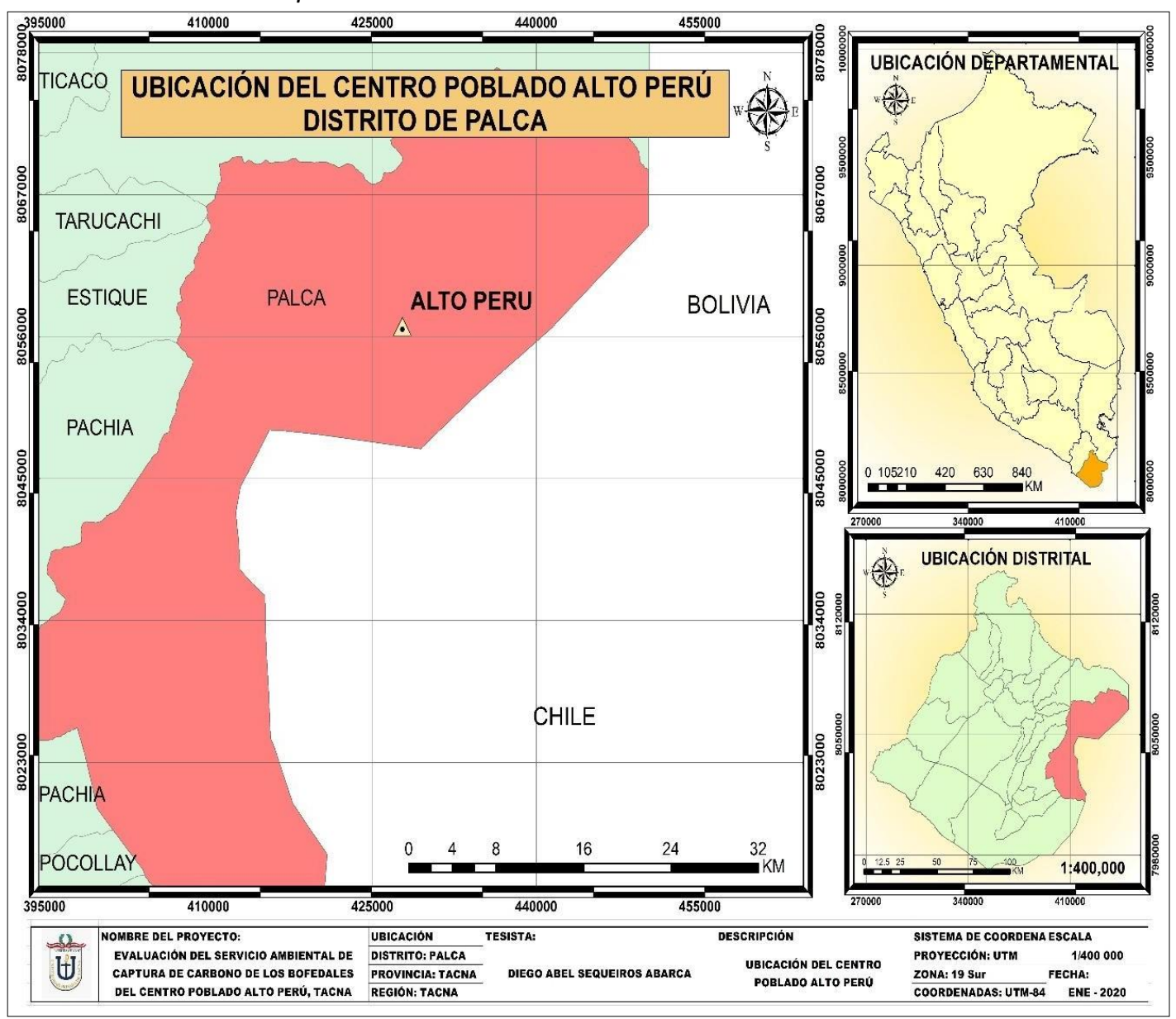

Fuente. Elaboración propia.

Para la ubicación y delimitación del área de estudio se calculó mediante el uso del sistema de información geográfica ArcGis versión 10.3, delimitándose el área correspondiente a zonas representativas de los bofedales del Centro Poblado Alto Perú, para este fin se descargaron imágenes satelitales Landsat 8, las cuales se usaron las franjas 4 de infrarrojo visible y 5 de infrarrojo cercano según la tabla 1, para determinar el Índice de Vegetación de Diferencia Normalizada (NDVI) con la fórmula 1. 


$$
\mathrm{NDVI}=\frac{(N I R-V I S)}{(N I R+V I S)}
$$

Donde

NDVI : Índice de vegetación de diferencia normalizada. NIR

: Reflectancia de banda 5, infrarrojo cercano.

VIS : Reflectancia de banda 4, infrarrojo visible.

Fuente : Rügnitz et al., (2009)

Tabla 1

Landsat 8 - Distribución de bandas

\begin{tabular}{lcc}
\hline \multicolumn{1}{c}{ Banda } & Ancho (um) & $\begin{array}{c}\text { Resolución } \\
\text { (m) }\end{array}$ \\
\hline Band 1 Coastal & $0,43-0,45$ & 30 \\
Band 2 Blue & $0,45-0,51$ & 30 \\
Band 3 Green & $0,53-0,59$ & 30 \\
Band 4 Red & $0,64-0,67$ & 30 \\
Band 5 NIR & $0,86-0,88$ & 30 \\
Band 6 SWIR1 & $1,57-1,65$ & 30 \\
Band 7 SWIR2 & $2,11-2,29$ & 30 \\
Band 8 Pan & $0,50-0,68$ & 15 \\
Band 9 Cirrus & $1,36-1,38$ & 30 \\
Band 10 TIRS1 & $10,6-11,19$ & 100 \\
Band 11 TIRS2 & $11,5-12,51$ & 100 \\
\hline
\end{tabular}

Nota. Adaptado de Ariza (2013).

De los resultados del NDVI, se establecieron 4 categorías de cobertura; zonas saturadas, zonas húmedas, zonas secas y suelo desnudo (López, 2015), que fueron convertidas a polígonos para obtener los porcentajes correspondientes a cada área. Los bofedales del Centro poblado Alto Perú, al ser longitudinal, se dividió en 2 zonas para una mejor interpretación, tal como se observa en la figura 2 y figura 3. 


\section{Figura 2}

Delimitación del área de estudio - Zona 01: Índice de vegetación de diferencia normalizada, (izquierda)

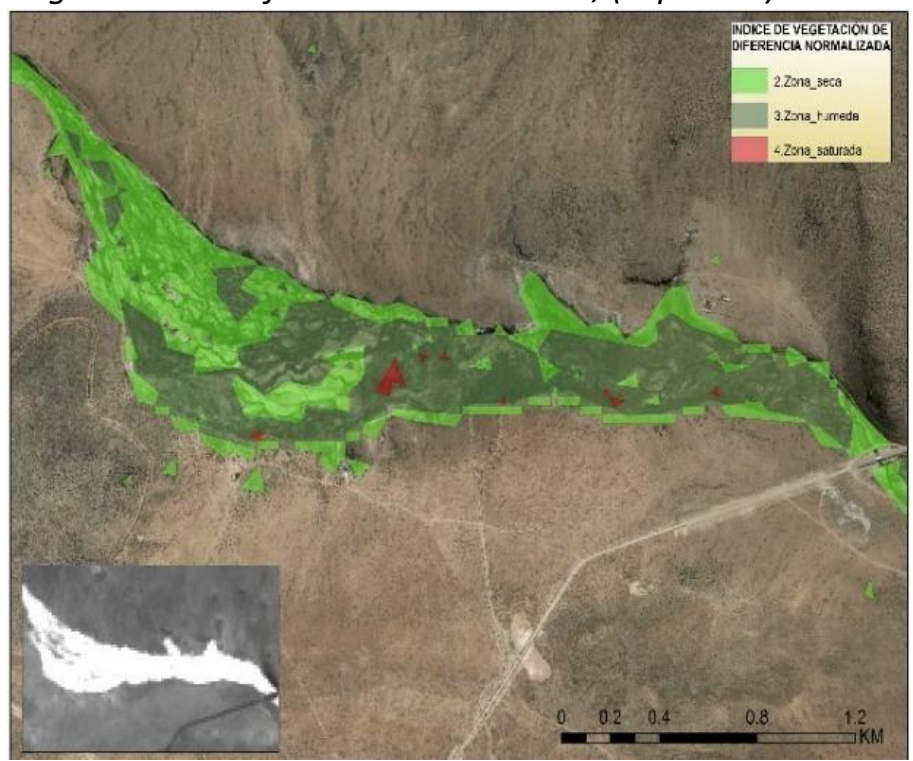

Nota. Elaboración propia

\section{Figura 3}

Delimitación del área de estudio - Zona 02: Índice de vegetación de diferencia normalizada, (derecha)

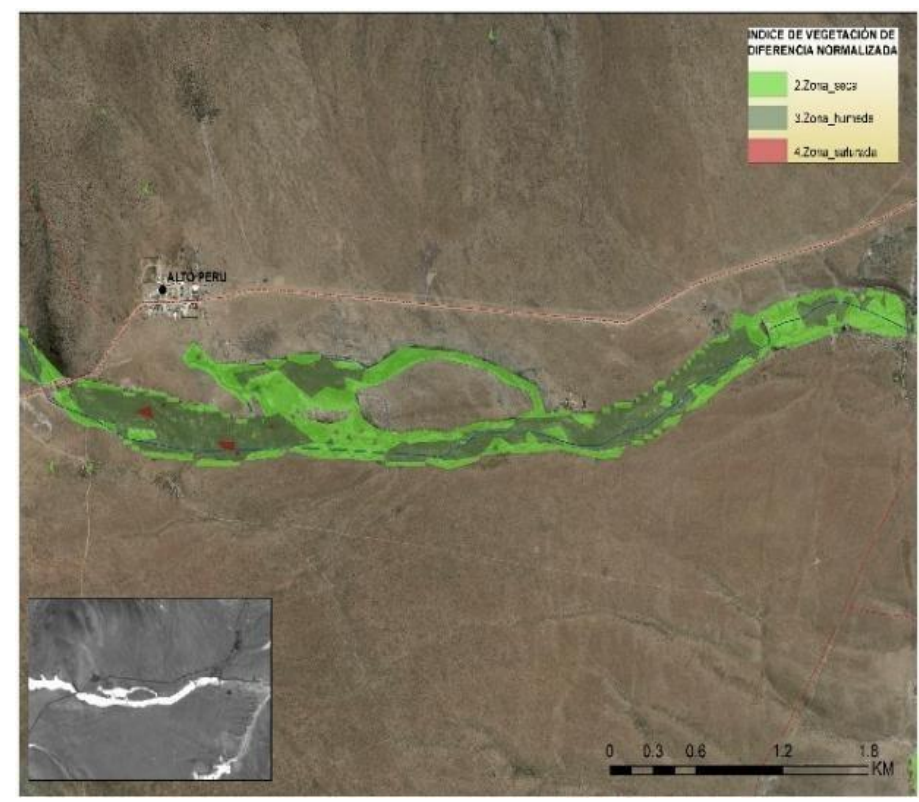

Nota. Elaboración propia.

Para el número de muestras se consideró las investigaciones de Palomino et al., (2007) quien menciona un número mínimo de 5 muestras por especie, así como Alvis (2017) quien determinó un 
número de 30 muestras para 882,54 ha Para este estudio, se estimó un total de 15 puntos de muestreo, las cuales fueron distribuidas de la siguiente manera; 8 para la zona 1 del bofedal (representa el $60 \%$ del área de estudio) y 8 para la zona 2 del bofedal (representa el $40 \%$ restante), dando así un total de 15 muestras para las 254,39 ha.

En el muestreo de vegetación herbácea, cada parcela se distribuyó con una equidistancia de por lo menos 100 m de separación entre ellas según la Guía de Inventario de Flora y Vegetación del Ministerio del Ambiente (2015) y para este caso se siguió la metodología planteada por Alvis (2017) y Rivera (2018), que mediante el uso del programa de ArcGis versión 10.3, se generaron grillas con dimensiones de $100 \times 100 \mathrm{~m}$, las cuales se sectorizaron por parcelas cuadradas equivalentes a dichas dimensiones mediante la herramienta denominada "create fishnet".

Con la finalidad de determinar la distribución de las muestras, se ubicó un punto céntrico en cada parcela generada en ArcGis, las cuales representan una posible unidad muestral, posteriormente mediante el programa MS Excel, se utilizó la función Aleatorio (Rivera, 2018), para determinar la ubicación de los 15 puntos en selección, los resultados fueron trasladados al mapa de ubicación y se determinó las coordenadas con la herramienta "Add XY Coordinates"; posteriormente se corrigió manualmente la ubicación de los puntos de muestreo para ubicar en sectores representativos del bofedal (zona húmeda y zona seca), según las figuras 4 y 5.

Figura 4

Parcelación mediante grillas de 100×100 m - Zona 01:

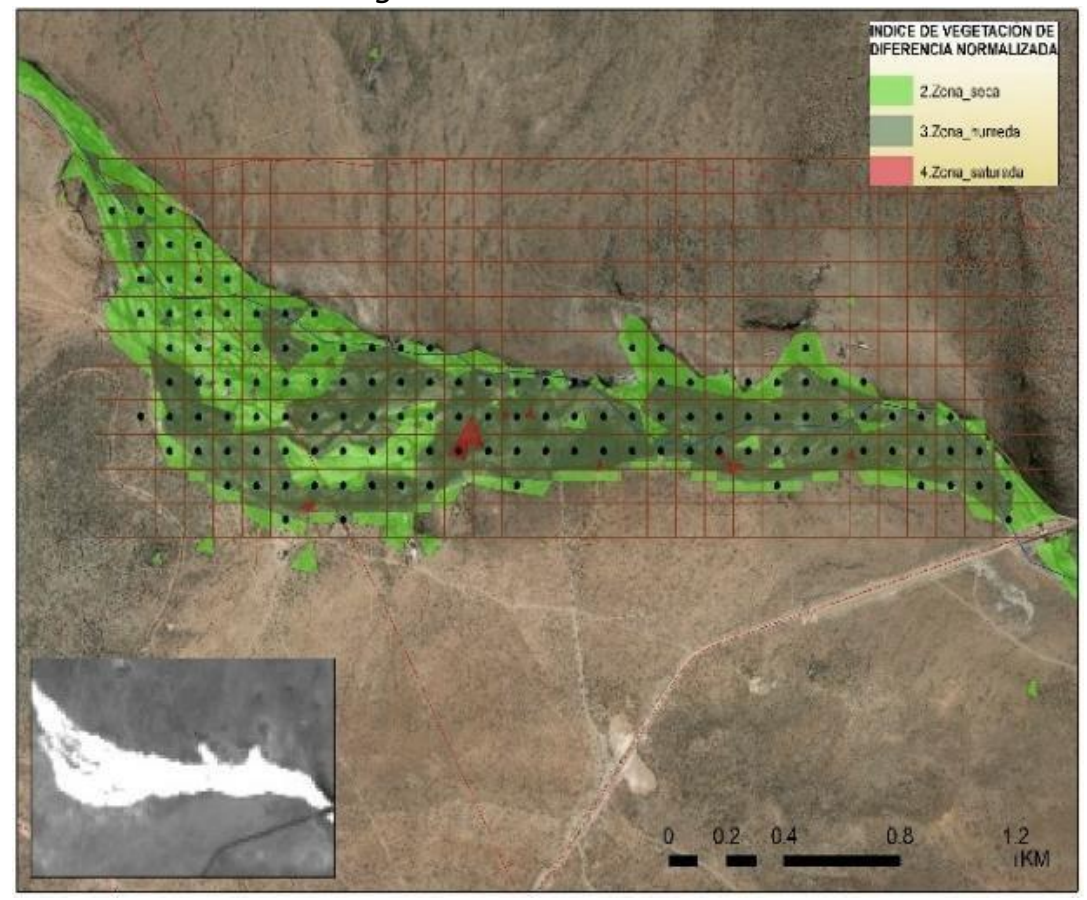

Nota. Elaboración propia. 


\section{Figura 5}

Parcelación mediante grillas de 100×100 m - Zona 02

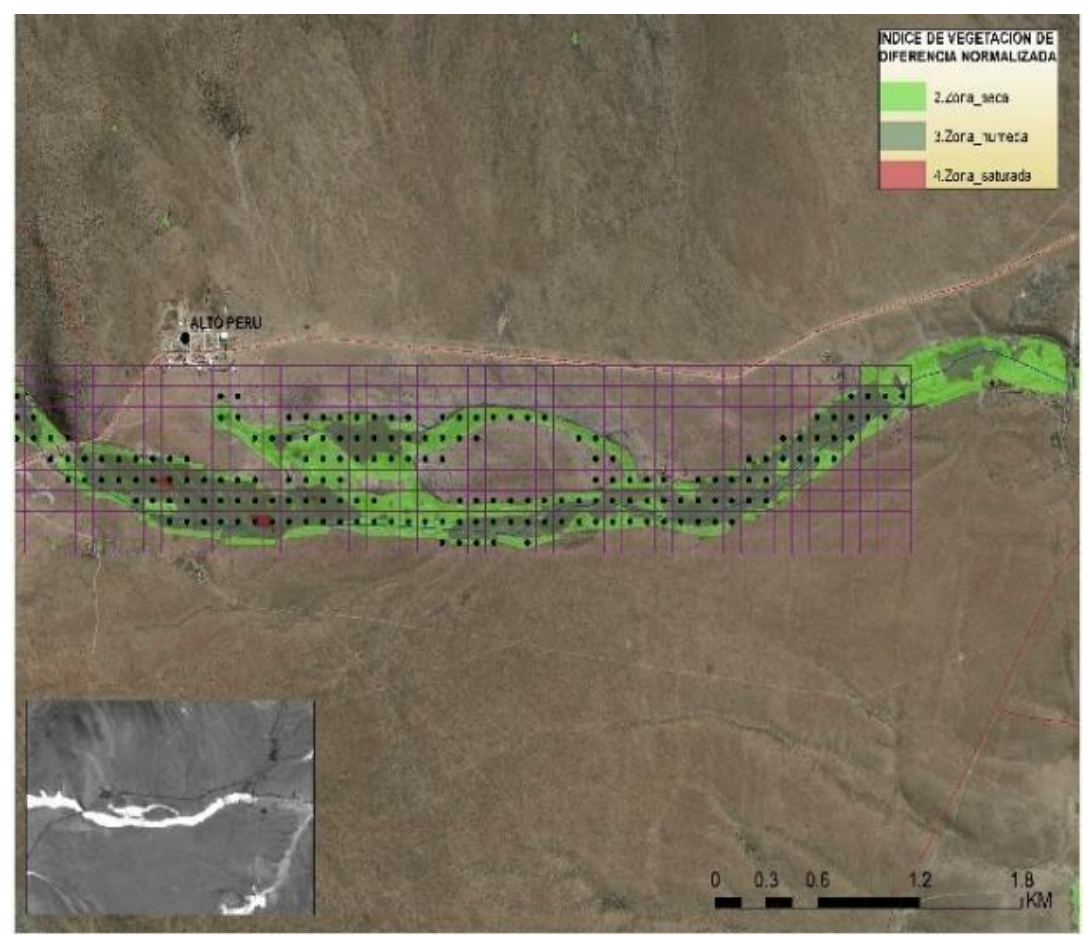

Nota. Elaboración propia

\section{Determinación de las reservas de carbono}

Para determinar las reservas de carbono en los bofedales es necesario conocer el contenido de carbono acumulado en la cobertura vegetal, así como el carbono acumulado bajo el suelo (raíz, materia muerta y suelo orgánico). Por tal motivo es que se describe la metodología proporcionada por el Centro Internacional de Investigación Agroforestal (ICRAF) para vegetación herbácea.

La toma de muestra de la cobertura vegetal en la parte aérea, Las coordenadas de los puntos de muestreo fueron tomadas en campo con un equipo GPS Garmin 10x, para encontrar la ubicación de los puntos, previas correcciones por accesibilidad, la metodología del muestreo fue el descrito por Alvis (2017) y Cochi et al., (2014), Mediante el uso de un radio muestreador de $10 \mathrm{~cm}$ de diámetro; se ubicó en el punto céntrico de cada tepe y se extrajo la materia vegetal viva con ayuda de una navaja; teniendo en consideración evitar las zonas heterogéneas, las cuales son características predominante de la biomasa herbácea (Pérez et al., 2015).

Para la Toma de muestra de la parte turba/necromasa, se empleó la metodología usada para la extracción de muestras de turba/necromasa debajo de la cobertura vegetal, se consideró lo planteado por Palomino (2007). Las muestras de la biomasa bajo el suelo (raíz y necromasa) fueron extraídas a 10 $\mathrm{cm}$ de profundidad, por debajo del radio muestreador ubicado en la parte aérea, mientras que, para las muestras de suelo, estas fueron extraídas a $30 \mathrm{~cm}$ debajo de cada punto mediante el uso de un barreno manual. En laboratorio, las muestras fueron separadas manualmente, la parte radicular, necromasa y el suelo orgánico. 
En el cálculo de la cantidad de carbono en la cobertura vegetal y turba, se empleó la guía metodológica del ICRAF, para determinar la cantidad de carbono presente en la vegetación, según el siguiente procedimiento:

a) Cálculo de la cantidad de carbono en la cobertura vegetal y turba (raíz necromasa) (Alvis (2017)

$$
\begin{aligned}
& C C B A=(P S M * \% C) * r^{2} * 100 \\
& C C B=(P S M * \% C) * r^{2} * 100
\end{aligned}
$$

Donde:

CCBA : Contenido de carbono en biomasa aérea $(\mathrm{g} / \mathrm{m} 2)$

CCBS : Contenido de carbono bajo el suelo $(\mathrm{g} / \mathrm{m} 2)$

PSM : Peso seco de la muestra (biomasa) (g).

\% C : Porcentaje de carbono determinado en laboratorio.

R : Radio del muestreador

b) Cálculo de la cantidad de carbono del suelo

Para el cálculo de la cantidad de carbono del suelo orgánico es necesario un muestreador, cuyo volumen sea conocido (barreno) para aplicar la fórmula 4 según Alvis (2017).

$$
=\pi * r^{2} * H B N
$$

Donde:

V : Volumen de la muestra (suelo orgánico/ suelo mineral) (m3).

$r \quad$ : Radio del muestreador $(\mathrm{m})$

HBN : Altura de la muestra sólida o fracción de la muestra encontrada (suelo orgánico/ suelo mineral) (m).

c) Cálculo de la densidad aparente del suelo(Alvis, 2017)

$$
D A=P S M / V
$$

Donde:

DA: Densidad aparente $(\mathrm{kg} / \mathrm{m} 3)$.

PSM: Peso seco de la muestra (suelo orgánico/suelo mineral) ( $\mathrm{kg}$ ).

V: Volumen de la muestra (suelo orgánico/suelo mineral) (m3). 
d) Cálculo de las reservas de carbono almacenado en suelo (Alvis, 2017)

$$
C C S O=(D A * H B N * \% C) / 100
$$

Donde:

DA : Densidad aparente $(\mathrm{g} / \mathrm{m} 3)$.

HBN : Altura de la muestra sólida (m).

\% $\quad$ : Resultado del porcentaje o fracción de carbono determinado en laboratorio.

Los resultados se presentan en $\mathrm{g} / \mathrm{m} 2$, para la conversión a TC/ha resultado total dividido entre 100

e) Cantidad de carbono en la biomasa de la vegetación no arbórea (Rügnitz et al., 2009)

$$
\triangle \mathrm{CBN}(\mathrm{TC} / \mathrm{ha})=(C C B A+C C B S+C C S O)
$$

Donde:

$\triangle C B N$ : Cantidad de carbono en la biomasa de vegetación no arbórea ( $\mathrm{t} C / \mathrm{ha}$ ).

CCBA : Cantidad de carbono en la cobertura vegetal ( $\mathrm{t} \mathrm{C} / \mathrm{ha}$ ).

CCBS : Cantidad de carbono turba/necromasa ( $\mathrm{C} / \mathrm{ha}$ ).

CCSO : Cantidad de carbono en suelo orgánico ( $\mathrm{t} \mathrm{C} / \mathrm{ha}$ ).

\section{Determinación de fracción de carbono}

Existen 3 métodos para determinar la fracción de carbono presente en la materia orgánica del suelo y residuos orgánicos vegetales y animales en cualquier grado de descomposición. Para el análisis de la materia orgánica en el suelo, se pueden utilizar métodos volumétricos, gravimétricos y colorimétricos, los más sencillos se basan en el consumo de un oxidante que actúa sobre el carbono, para posteriormente determinarlo por colorimetría o volumetría.

El método de Walkey y Black, indica que la materia orgánica es oxidada con una mezcla de $\mathrm{K}_{2} \mathrm{CrO}_{7}$ más $\mathrm{H}_{2} \mathrm{SO}_{4}$. La dilución concentrada se calienta con $\mathrm{H}_{2} \mathrm{SO}_{4}$ y $\mathrm{K}_{2} \mathrm{CrO}_{7}$ y es la fuente exclusiva de calor. El exceso de $\mathrm{K}_{2} \mathrm{CrO}_{7}$ es determinado por titulación con $\mathrm{FeSO}_{4}$ o con $\left(\mathrm{NH}_{4} \mathrm{Fe}\right)_{2}\left(\mathrm{SO}_{4}\right)_{3}$. Este método, proporciona una estimación fácilmente oxidable de carbono orgánico y es usado como una medida de carbono orgánico total. El porcentaje de materia orgánica es igual al porcentaje de carbono orgánico, multiplicado por el factor de 1,724 porque la materia orgánica contiene el $58 \%$ de carbono (Palomino \& Cabrera, 2007).

El procedimiento consiste en calcular las muestras en peso seco de las especies vegetales y muestras de suelo tamizadas con una malla de $2 \mathrm{~mm}$, luego moler las muestras de vegetación mediante el uso de un molino. Pesar 0,2 g de muestra y llevar a un frasco de Erlemeyer de $250 \mathrm{ml}$, luego agregar a 
las muestras $20 \mathrm{~mL}$ de solución Dicromato de potasio $\left(\mathrm{K}_{2} \mathrm{Cr}_{2} \mathrm{O}_{7}\right) 2 \mathrm{~N}$, Posteriormente, agregar $10 \mathrm{ml}$ de Ácido sulfúrico concentrado $\left(\mathrm{H}_{2} \mathrm{SO}_{4}\right)$ concentrado, mezclar para homogeneizar la solución. Reposar las muestras de 2 a 3 horas, seguidamente enrasar a $100 \mathrm{ml}$ con agua deshionizada (previamente hacer un blanco). Finalmente titular con solución Sulfato ferroso Amoniacal 0,2 N, agregando gotas de indicador Difenil amina sulfúrica 1 \% ( 3 gotas $/ 20 \mathrm{~mL}$ ), hacer los cálculos según la fórmula 7, propuesta por Palomino (2007).

$$
\% M O=M * \frac{1.724 * 0.4 * N s f a *(V b-V m)}{\text { Peso inicial de la muestra }(g)}
$$

Donde:

M : Es la molaridad de la solución de $\mathrm{FeSO} 4$

0.4 : Es el factor equivalente del peso del carbón

$\mathrm{Vb} \quad$ : Es el volumen de la solución Ferrosa amoniacal requerido para el blanco (ml)

Vm : Es el volumen de la solución Ferrosa amoniacal requerido para la muestra $(\mathrm{ml})$

Nsfa : Normalidad de la solución ferrosa amoniacal = 4 / Vsfabk (Volumen de la solución sulfato ferrosa amoniacal del blanco)

\section{Resultados}

\section{Fracción de carbono}

Los valores de fracción de carbono orgánico son necesarios para la obtener el resultado del carbono orgánico total, los resultados se analizaron tres muestras por punto de muestreo descritos en la tabla 2, en ella se observa que la fracción de carbono orgánico promedio en los puntos de muestreo de la cobertura vegetal, según el mapa de NDVI y la fórmula siete, en zonas secas es de $42,04 \%$, mientras que en zonas húmedas es del $45,61 \%$, obteniéndose así un promedio total de $43,83 \%$ de carbono almacenado en la toda la cobertura del bofedal. Por otro lado la fracción de carbono orgánico promedio de las muestras en la turba/necromasa, según el mapa de NDVI y a la fórmula $\mathrm{N}^{\circ} 07$, en zonas secas es de $33,17 \%$, mientras que en zonas húmedas es del $36.26 \%$, obteniendo así un promedio total de $34,72 \%$ de carbono almacenado bajo la cobertura vegetal del bofedal (turba).

La fracción de carbono orgánico promedio de las muestras en el suelo de acuerdo al mapa de NDVI y a la formula $\mathrm{N}^{\circ} 7$, en zonas secas es de $12,26 \%$, mientras que en zonas húmedas es del $12,85 \%$, obteniendo así un promedio total de 12,56 \% de carbono almacenado en el suelo orgánico del bofedal, a una profundidad de $30 \mathrm{~cm}$. 


\section{Tabla 2}

\begin{tabular}{cccc}
\multicolumn{4}{c}{ Fracción de carbono en la cobertura vegetal, turba/necromasa y Suelo } \\
\hline Muestra & $\begin{array}{c}\text { Cobertura } \\
\text { vegetal (\%) }\end{array}$ & $\begin{array}{c}\text { turba/necromasa } \\
\text { (\%) }\end{array}$ & $\begin{array}{c}\text { Suelo } \\
\text { (\%) }\end{array}$ \\
\hline ZH-001 & 53,70 & 50,18 & 24,70 \\
ZS-002 & 40,77 & 45,08 & 30,38 \\
ZS-003 & 39,00 & 39,98 & 13,48 \\
ZH-004 & 45,63 & 43,71 & 14,27 \\
ZH-005 & 37,83 & 34,10 & 9,56 \\
ZS-006 & 44,88 & 29,79 & 10,82 \\
ZS-008 & 47,65 & 44,69 & 6,27 \\
ZH-009 & 41,75 & 36,85 & 13,56 \\
ZS-001 & 43,90 & 20,97 & 19,60 \\
ZS-002 & 46,26 & 10,19 & 3,06 \\
ZH-003 & 52,53 & 33,32 & 11,37 \\
ZH-004 & 38,02 & 39,40 & 12,78 \\
ZS-005 & 38,22 & 43,12 & 6,74 \\
ZH-006 & 49,78 & 16,27 & 3,68 \\
ZS-007 & 43,51 & 31,56 & 7,76 \\
& & & \\
\hline
\end{tabular}

Nota. Elaboración propia. ZS= zona seca, ZH= zona húmeda

\section{Tabla 4}

Resumen de fracción de carbono

\begin{tabular}{lccc}
\hline Tipo de Muestra & $\begin{array}{c}\text { Cobertura } \\
\text { vegetal (\%) }\end{array}$ & $\begin{array}{c}\text { Turba/necromasa Suelo orgánico } \\
\text { (\%) }\end{array}$ & (\%) \\
\hline Zona seca & & & \\
Zona húmeda & 42,04 & 33,17 & 12,26 \\
Total & 45,61 & 36,26 & 12,85 \\
\hline
\end{tabular}

Nota. Elaboración propia

\section{Resultados del carbono total almacenado por tipo de muestra}

Con los valores obtenidos, por el peso seco de las muestras y la fracción de carbono orgánico, se obtuvo el resultado del carbono total almacenado en los bofedales del CP Alto Perú, donde los resultados son expresados en toneladas de carbono por hectárea ( $\mathrm{TnC} / \mathrm{ha}$ ) para cada punto de muestreo 
descritos a continuación.

El carbono orgánico total de las muestras colectadas en la cobertura vegetal, de acuerdo a la fórmula $\mathrm{N}^{\circ} 02$, en zonas secas es de $12,30 \mathrm{TnC} / \mathrm{ha}$, mientras que en zonas húmedas es del $18.36 \mathrm{TnC} / \mathrm{ha}$, obteniendo así promedio total de 15,33 TnC/ha almacenado en la parte aérea del bofedal.

El carbono orgánico total de las muestras colectadas en la turba/necromasa, de acuerdo a la fórmula $\mathrm{N}^{\circ} 02$, en zonas secas es de 22,52 TnC/ha, mientras que en zonas húmedas es del 29,89 TnC/ha, obteniendo así un promedio total de $26,20 \mathrm{TnC} / \mathrm{ha}$ almacenado en la turba del bofedal (turba).

El carbono orgánico total de las muestras colectadas en el suelo orgánico, de acuerdo a las fórmulas $\mathrm{N}^{\circ}$ 03, 04 y 05, en zonas secas es de 130,49 TnC/ha, mientras que en zonas húmedas es del 100.80 TnC/ha, obteniendo así un promedio total de 115,65 TnC/ha almacenado en el suelo orgánico bofedal, a una profundidad de $30 \mathrm{~cm}$.

\begin{tabular}{cccc}
$\begin{array}{l}\text { Tabla } 5 \\
\text { Carbono total en la cobertura vegetal, turba/necromasa y Suelo }\end{array}$ \\
\hline Muestra & $\begin{array}{c}\text { Cobertura vegetal } \\
\text { TnC/harba/necromasa }\end{array}$ & $\begin{array}{l}\text { suelo orgánico } \\
\text { TnC/ha }\end{array}$ \\
\hline ZH-001 & 20,30 & 41,93 & 54,04 \\
ZS-002 & 12,17 & 34,98 & 345,71 \\
ZS-003 & 10,09 & 22,27 & 138,16 \\
ZH-004 & 17,25 & 44,35 & 214,04 \\
ZH-005 & 17,31 & 30,53 & 70,41 \\
ZS-006 & 11,61 & 13,63 & 86,10 \\
ZS-008 & 14,22 & 27,56 & 89,71 \\
ZH-009 & 15,78 & 34,45 & 105,77 \\
ZS-001 & 11,48 & 25,45 & 147,78 \\
ZS-002 & 13,80 & 9,73 & 8,82 \\
ZH-003 & 21,95 & 25,19 & 30,98 \\
ZH-004 & 15,13 & 22,73 & 193,73 \\
ZS-005 & 12,93 & 28,31 & 96,85 \\
ZH-006 & 20,80 & 10,03 & 36,65 \\
ZS-007 & 12,12 & 18,21 & 130,79 \\
\hline
\end{tabular}

Nota. Elaboración propia

\section{Carbono total almacenado en los bofedales de Alto Perú}

Con los valores obtenidos del carbono total, almacenado por el tipo de muestra, se obtiene la cantidad de carbono total que almacenan los bofedales del CP Alto Perú; estos resultados son expresados en toneladas de carbono por hectárea ( $\mathrm{Tn} \mathrm{C} / \mathrm{ha}$ ), tal como se observa en la tabla 6 
Tabla 6

Resumen del carbono total en la cobertura vegetal, turba/necromasa y suelo orgánico

\begin{tabular}{lcccc}
\hline $\begin{array}{l}\text { Tipo de } \\
\text { Muestra }\end{array}$ & $\begin{array}{c}\text { Cobertura vegetal } \\
\text { Tn C/ha }\end{array}$ & $\begin{array}{c}\text { Turba/Necromasa Suelo Orgánico } \\
\text { Tn C/ha }\end{array}$ & $\begin{array}{c}\text { Carbono total } \\
\text { almacenado }\end{array}$ \\
\hline Zona seca & 12,30 & 22,52 & 130,49 & Tn C/ha \\
Zona húmeda & 18,36 & 29,89 & 100,80 & 157,18 \\
Promedio & 15,33 & 26,20 & 115,18 & \\
\hline
\end{tabular}

Nota. Elaboración propia. (a) Suma de promedios

El carbono orgánico total almacenado en el bofedal del Centro Poblado Alto Perú es el resultado de los sumatoria de los promedios que resulto en $157,18 \mathrm{Tn} \mathrm{C} / \mathrm{ha}$.

\section{Evaluación de dióxido de carbono fijado}

De acuerdo a la fórmula presentada por Palomino (2007) y Alvis (2017), para la cuantificación de dióxido de carbono almacenado, se multiplica el valor obtenido del carbono orgánico total, expresado en $\mathrm{TnC} /$ ha por un valor constante $(k r)$ de 44/12, dando como resultado una fijación de 576.22 TnCO2/ha en los bofedales del Centro Poblado Alto Perú.

Se realizó un análisis estadístico de análisis de varianza para verificar qué tipo de muestra aporta mayor carbono orgánico total, entre la cobertura vegetal, turba/necromasa y el suelo orgánico encontrándose diferencias significativas ( $p$-valor $=0,000$ ) en el aporte de carbono por tipo de muestra, posteriormente para ver qué tipo de muestra aporta más, se realizó la prueba post hoc de Tukey encontrándose que la cobertura vegetal y la turba/necromasa aporta menos carbono orgánico total, a diferencia del suelo orgánico, según la significancia de cada tipo de muestra al $95 \%$ de confianza.

\section{Discusión}

\section{Fracción de carbono en la cobertura vegetal}

Al hallarse una diferencia del 3,57 \% entre las fracciones de zonas secas y húmedas del bofedal, evidencia que no existe gran diferencia en la fracción de carbono de la parte aérea. Los valores obtenidos $(42,04$ y $45,61 \%)$ son similares a valores obtenidos en investigaciones previas, tales como lo reportado por Alvis (2017) en humedales alto andinos, reportando $49 \%$ de fracción de carbono, mientras que en la investigación en humedales costeros, Palomino (2007) reportó 47\% de carbono.

La diferencia entre los resultados se puede explicar debido a la influencia de diferentes factores, destacando la predominancia de especies vegetales presentes en el bofedal. Alvis (2017) muestra una predominancia de Philloscirpus desertícola (24/30), D. muscoides (12/30), Werneria sp. (13/30), 
mientras que Mamani (2015), en el inventario florístico de los bofedales, muestra una predominancia por familias de Juncaceae $(15,75 \%)$ y Asteraceae $(13,08 \%)$.

Los valores en la fracción de carbono en bofedales, a diferencia de los fracciones en los humedales costeros se explican, por aspectos climáticos de la zona de estudio, así lo explica Alvis (2017), refiriéndose a la velocidad del viento, ya que a una mayor intensidad, existe una mayor circulación del aire pobre en $\mathrm{CO} 2$ de las capas límite de las hojas. Otro aspecto influyente es la elevada altitud de la zona de estudio (+ $4100 \mathrm{msnm}$ ), ya que al encontrarse en altitudes más elevadas, que incidiría en una disminución de la concentración de oxígeno y el aumento de la concentración de otros gases como el $\mathrm{CO} 2$, incrementando así la fracción de carbono presente en la cobertura vegetal.

\section{Fracción de carbono en la Turba/necromasa}

Existe una diferencia del 3,09 \% entre los parches de bofedal seco y húmedo del bofedal, la reducida diferencia en cuanto a la fracción de carbono obtenido en ambas partes puede explicarse por Mamani (2015), que menciona que en los bofedales hidromórficos, compuestos principalmente por Oxichloe andina y Distichia muscoides, la turba puede llegar hasta un metro de profundidad, explicándose así, la poca diferencia entre los porcentajes existente entre zonas secas y húmedas debido a que ambos presentarían turba con semejantes niveles profundidades y no encontrarse una interferencia con la siguiente muestra de suelo orgánico. Por otro lado los valores obtenidos $(33,17$ y $36,26 \%)$ difieren de los porcentajes obtenidos por Alvis (2017) en humedales alto andinos (42,53\%), mientras que Pérez (2015) quien reporta 34,44 \% en fracción de carbono. Esta diferencia podría darse por diversos motivos, entre ellos, la profundidad de la turba con respecto al suelo orgánico, ya que en algunos puntos de muestreo la turba llegó a profundidades de $20 \mathrm{~cm}$, mientras que en otros puntos de muestreo sobrepasaron los $30 \mathrm{~cm}$ de profundidad.

\section{Fracción de carbono en el suelo orgánico}

Existe una diferencia del 0,58 \% entre las fracciones de zonas secas y húmedas, indicando que no existe diferencia significativa en cuanto a la fracción del suelo orgánico. Los valores obtenidos (12,26 y $12,85 \%)$, son inferiores a los obtenidos en investigaciones por Alvis (2017), en humedales alto andinos con 43,05\%, mientras que en humedales costeros, Palomino (2007) reportó 3 \%. Por otro lado según Hribljan et al., (2015), en los bofedales del pueblo de Manasaya - Bolivia muestra porcentajes cercanos con 23,90 \% para suelo orgánico del bofedal. La diferencia existente, podría ser explicada por la profundidad de la turba en el correspondiente punto de muestreo, esto debido a que en algunos puntos de muestreo, con profundidades menores a $30 \mathrm{~cm}$ de turba, se obtiene porcentajes de carbono con valores bajos como $3,06 \%$, mientras que en otros, la turba que sobrepasa los $30 \mathrm{~cm}$ de profundidad, resultando valores cercanos a 30,38\%. En ese sentido Alvis (2017) mencionan que del total del dióxido de carbono captado por el proceso fotosintético el $20 \%$ pasa a la capa inferior de la turba, del cual se va perdiendo aún más por el proceso de descomposición anaeróbica en el catotelmo. Por tal motivo a medida que se va incrementando la profundidad el carbono total va disminuyendo y acercándose a la siguiente capa denominado "suelo mineral". 


\section{Carbono total almacenado en la cobertura vegetal}

Al analizar el carbono total en la cobertura vegetal se encontró 6,06 TnC/ha de diferencia del total almacenado en la cobertura vegetal entre zonas secas y húmedas, esta diferencia es explicada por Hernández (2010) quien menciona que el estado de conservación de la cobertura vegetal es una variable crítica para la regulación del ciclo del carbono; deduciéndose de tal forma que un bofedal con buen estado de conservación, incrementaría la biomasa presente en proporción de zonas secas y húmedas, ante ello el estado de conservación explicaría el equilibrio entre la emisión de dióxido de carbono y/o metano a la atmosfera producido por la descomposición de la materia orgánica y la captación de dióxido de carbono por procesos fotosintéticos. Los valores obtenidos (12,30 y 18,36 TnC/ha), son ligeramente superiores a los evidenciados por Alvis (2017) quien reporta 4,77 TnC/ha para humedales alto andinos, mientras que Medrano et al., (2012) reportando 5,00 TnC/ha para bofedales y totorales, así mismo son ligeramente inferiores a lo encontrado por Palomino (2007) para humedales costeros $(20,10 \mathrm{TnC} / \mathrm{ha})$ para la cobertura vegetal.

Como se mencionó anteriormente, la diferencia entre los resultados se puede explicar debido a diferentes factores entre ellos, la predominancia de familias vegetales presentes en el bofedal y a las especies muestreadas en esta investigación (D. muscoides., Oxyclhoe andina). La diferencia con Medrano et al., (2012), se puede explicar debido a que en su investigación en el ecosistema de bofedal, se colectaron muestras de tres especies vegetales: Plantago tubulosa, Eleocharis albibracteata y Limosella australis, según el Plan Maestro del ACRVM (2012), dichas especies se caracterizan por ser acuáticas, encontradas en ambientes semi o permanentemente inundables, cuya característica no es similar a formaciones almohadilladas como de la familia juncaceae (Distichia muscoides, Distichia sp, Oxychloe andina).

\section{Carbono total almacenado en la Turba/necromasa}

Existe una diferencia de 7,37 TnC/ha del total almacenado en turba/necromasa entre zonas secas y húmedas, al igual que con la parte aérea, esta diferencia puede explicarse debido al estado de conservación de la cobertura vegetal, porque el estado de salud de la vegetación, también influiría en el ciclo del carbono de la fase de captación a sumidero del mismo. Los valores obtenidos $(22,52$ y 29,89 $\mathrm{TnC} / \mathrm{ha}$ ), dichos valotes son superiores a los obtenidos por Alvis (2017) en humedales alto andinos $(19,72 \mathrm{TnC} / \mathrm{ha}$ ) y Palomino (2007) para humedales costeros (20,10 TnC/ha) para la parte de suelo y raíz del humedal. Existen diferentes variables que pueden explicar la diferencia entre los resultados con los humedales costeros y alto andinos para la parte de la turba, uno de los factores a considerar es la predominancia de familias vegetales presentes en el bofedal y a las especies muestreadas en esta investigación (D. muscoides., Oxyclhoe andina). Alvis (2017) sostiene que, la vegetación de tipo perenne tienen a presentar una renovación foliar más lenta y esto resultaría en un tiempo de vida más largo de la parte aérea y una acumulación continúa en la turba; al tener una acumulación continua y bajos niveles de descomposición, la acumulación de la biomasa de abajo de la parte aérea se incremente significativamente y esto explicaría los altos niveles en el carbono orgánico total. 


\section{Carbono total almacenado en el suelo orgánico}

Se encontró una diferencia de $29,2 \mathrm{TnC}$ /ha entre las muestras provenientes de zonas húmedas y secas, evidenciando que existe una mayor cantidad de carbono almacenado en zonas secas, esta diferencia entre el resultados puede explicarse principalmente por la profundidad en la que se tomó la muestra en relación con la profundidad total de la turba, ya que como se mencionó anteriormente en ciertos puntos de muestreo la turba no sobrepasaba $10 \mathrm{~s} 30 \mathrm{~cm}$ de profundidad, mientras que en otros puntos de muestreo, dicha turba sobrepasa los $30 \mathrm{~cm}$ de profundidad, por consiguiente incrementa la fracción de carbono, pero disminuyendo la densidad aparente de la muestra. Al respecto Palomino (2007), encontró valores de 9,57 TnC/ha, mientras que Alvis (2017) mostró 218,28 TnC/ha. Valores más próximos han sido reportados por Calderón et al., (2013) en humedal andino de una reserva con 112,20 $\mathrm{TnC} / \mathrm{ha}$. La diferencia encontradas, puede explicase debido a diversos factores, además de los ya planteados anteriormente (profundidad de la turba, especie muestreada, profundidad de la toma de muestra, estado de salud de la cobertura vegetal). Así lo explica Alvis (2017), quien su vez cita a Marín et al., (2011) ya que en su investigación, plantea otras variables influyentes en las reservas de carbono en el suelo como; la edad del bofedal, porque algunos bofedales, al ser más antiguos suelen tener turbas a mayor profundidad, significando así un incremento en la profundidad de las muestras de suelo orgánico, llegando así a contener mayores reservas. Otra variable a tener en cuenta es la densidad aparente del suelo, que al contener una mayor densidad, incrementaría significativamente los valores de carbono en el suelo.

\section{Carbono total almacenado en los bofedales de Alto Perú}

De acuerdo a la metodología descrita por Hernández (2010), la sumatoria de las reservas entre la cobertura vegetal $(15,33 \mathrm{TnC} / \mathrm{ha})$, la turba/necromasa $(26,20 \mathrm{TnC} / \mathrm{ha})$ y el suelo orgánico (115,65 $\mathrm{TnC} / \mathrm{ha}$ ) resultó en una reserva total de 157,18 $\mathrm{TnC} / \mathrm{ha}$. El valor encontrado difiere a lo reportado por Alvis (2017) con 242,77 TnC/ha, Palomino (2007) con 20,1 TnC/ha, Medrano (2012) con 684,58 TnC/ha y Calderón et al., (2013) con 112,20 TnC/ha su explicación estriba en el tipo de muestra que determinaría una mayor o menor cantidad en las reservas de carbono de un humedal, es la encontrada en los suelos ya que, tomando como ejemplo los resultados de esta investigación representa el $73,57 \%$ del total encontrado entre los tres tipos de muestras.

\section{Dióxido de carbono fijado}

Los resultados de la cuantificación del servicio ambiental de captura de dióxido de carbono; $576,22 \mathrm{TnCO} / \mathrm{ha}$, son superiores a los presentados en humedales costeros por Palomino (2007), con valores de 73,70 TnCO2/ha; por otro Alvis (2017) presenta valores de 901,28 TnCO2/ha. Los valores comparados con los encontrados por otras investigaciones es explicada por Arellano et al., (2013) quien menciona que existen factores como la topografía, la salinidad, el microclima y la estacionalidad que influyen en la captura de carbono atmosférico. Los bajos niveles de precipitación que ocurren en la zona de estudio, podría ser un factor a tener en cuenta entre los niveles de fijación de dióxido de carbono, de acuerdo al SENAMHI se registraron niveles de precipitación de $363 \mathrm{~mm}$ durante el año 2019, caracterizándose así por ser un año con mayor precipitación a la media anual de 352,94 mm Como bien 
menciona Blanco et al., (2004) quien afirma que los niveles de hidratación de una turbera es un factor que sirve conocer la diferencia entre el acrotelmo y catotelmo respecto a la profundidad en cuestión de cada capa; es decir haber mayor hidratación en la turbera, la capa superior (acrotelmo) reduce su espesor, mientras que la capa inferior (catotelmo) al estar más saturada se va incrementado; acercarse así el nivel freático a la superficie. Una de las principales características del acrotelmo es la presencia de bacterias aeróbicas que aceleran la descomposición de la materia orgánica, y como lo menciona Hernández (2010), en el "ciclo del carbono en humedales", la descomposición de la materia orgánica produce la liberación de $\mathrm{CO} 2$ a la atmosfera; explicándose así, que debido a los bajos niveles de precipitación que se registraron durante el 2019 influirían en la recarga del acuífero y que traería consigo, un acrotelmo con mayor espesor, por consiguiente tendríamos bajos niveles en el carbono orgánico total almacenado en el tejido vegetal y/o necromasa, y bajos niveles de fijación de dióxido de carbono atmosférico.

\section{Conclusiones}

La fracción de carbono por tipo de muestra, para la cobertura vegetal, resulto que la parte aérea es $44,23 \%$, para la turba (necromasa/raíz) es $34,61 \%$ y para el suelo orgánico es $12,53 \%$; demostrando así que la parte aérea tendría un mayor potencial para la fijación de carbono en las juncáceas Oxichloe andina y Distichia muscoides.

El carbono total almacenado por tipo de muestra; para la cobertura vegetal es $15,33 \mathrm{TnC} / \mathrm{Ha}$, para la turba/necromasa es de $26,20 \mathrm{Tn} \mathrm{C} / \mathrm{Ha}$ y para el suelo orgánico es 115,65 Tn C/Ha; dando un total de 157,18 Tn C/Ha para los bofedales del Centro Poblado Alto Perú. Del tipo de muestra, se puede evidenciar que las mayores reservas de carbono se dan en el suelo orgánico, siendo un factor determinante la densidad aparente del suelo.

Las diferencias en el carbono total almacenado en zonas secas y húmedas son; para la cobertura vegetal 12,30 y $18,36 \mathrm{Tn} \mathrm{C} / \mathrm{ha}$, para la turba/necromasa 22,52 y $29,89 \mathrm{Tn} \mathrm{C} / \mathrm{Ha}$ y para el suelo orgánico es 130,49 y $100,80 \mathrm{Tn} \mathrm{C} / \mathrm{Ha}$; demostrando que existe diferencias entre el potencial de almacenamiento de carbono en zonas húmedas y secas del bofedal, de acuerdo a los resultados del NDVI.

En cuanto al potencial de captura de dióxido de carbono se obtuvo 576,22 Tn CO2/Ha; demostrando así, el gran potencial como servicio ambiental que tienen los bofedales del Centro Poblado Alto Perú, en comparación con otras investigaciones similares.

\section{Referencias Bibliográficas}

ACRVM, P. M. (2012). Plan Maestro Área del Conservación Regional Vilacota Maure. Tacna. 
Alvis, T. (2017). Almacenamiento de carbono en los humedales altoandinos del centro poblado Chalhua, Caylloma - Arequipa. Arequipa.

Arellano, E., Meza, F., Miranda, M., \& Camaño, A. (2013). El cuidado de los Humedales y su rol en el secuestro de carbono. Arauco: Investigadores del Centro de Cambio Climático Global UC.

Ariza, A. (2013). Descripción y Corrección de Productos Landsat 8 LDCM (Landsat Continuity Mission). Bogotá: Instituto Geográfico Agustín Codazzi.

Blanco, D., \& Balze, V. (2004). Los turbales de la Patagonia, bases para su inventario y la conservación de su biodiversidad. Buenos Aires: Wetlands international, publicación 19.

Calderón, M., Romero, H., Cuesta, F., Pinto, E., \& Báez, S. (2013). Monitoreo de contenidos y flujos de carbono en gradientes altitudinales altoandinos. Quito: condesan/cosude.

Cochi, N., Prieto, G., Rojas, A., Celso, A., Condori, B., \& Cazasola, J. (2014). Metodología para evaluar el potencial productivo y la dinámica socioecológica de la ganadería en bofedales altoandinos. La Paz, Bolivia.

Flores, M., Alegría, J., \& Granada, A. (2005). Diversidad floristica asociada a las lagunas andinas Pomacocha y Habascocha, Junín, Perú. Scielo - Perú.

Franco, L., Delgado, J., \& Andrade, G. (2012). Factores de la vulnerabilidad de los humedales altoandinos de Colombia al cambio climático global. Revista Colombiana de Geografía.

Hernández, M. E. (2010). Suelo de los humedales como sumideros de carbono y fuentes de metano. Terra Latinoamericana, 28.

López, E. (2015). Estudio de Biodiversidad de los Bofedales de Huaytire, Challapujo, Livicani, Japopunco y Surapata en el Centro Poblado Huaytire. Tacna: Gobierno Regional de Tacna.

Mamani, Y. J. (2015). Estado actual, diversidad florística y capacidad de carga del bofedal de Ancomarca del distrito de Palca, departamento de Tacna - Perú. Tacna: Universidad Nacional Jorge Basadre Grohmann.

Medrano, R., Chupán, L., \& Vila, M. (2012). Almacenamiento de carbono en especies predominantes de flora en el lago Chinchaycocha. Apunt. cienc, 02-02.

Palomino, D., \& Cabrera, C. (2007). Estimación del servicio ambiental de captura de CO2 en la flora de los humedales de Puerto Viejo. Revista del Instituto de Investigación FIGMMG.

Rivera, E. (2018). Evaluación del potencial almacenamiento de carbono en la especie Stipa ichu (Ichu) en la microcuenca Chaclatacana - Huancavelica. Lima: Universidad Nacional Federico Villareal.

Rügnitz, M., Chacón, M., \& Porro, R. (2009). Guía para determinación de carbono en pequeñas propiedades rurales. Centro Mundial Agroforestal - ICRAF.

\section{Agradecimientos}

Los autores agradecen a la profesional de la escuela Profesional de Ingeniería Civil de la Universidad Privada de Tacna, por su apoyo en la gestión de la investigación. 